\title{
LA IMPORTANCIA DE LOS NICHOS DE MERCADO. UN ESTUDIO DE CASO DEL MAÎZ AZUL Y DEL MAÍZ PARA POZOLE EN MÉXICO
}

\author{
THE IMPORTANCE OF NICHE MARKETS. A CASE STUDY OF BLUE AND \\ POZOLE-MAKING MAIZE IN MÉXICO
}

\author{
Jon Hellin $^{1 *}$, Alder Keleman ${ }^{2}$, Damaris López ${ }^{1}$ Laura Donnet $^{1}$ y Dagoberto Flores ${ }^{1}$
}

\begin{abstract}
${ }^{1}$ Centro Internacional de Mejoramiento de Maíz y Trigo. Km 45 carretera México-Veracruz. El Batán. 56130, Texcoco, Edo. de México. ${ }^{2}$ Faculty of Forestry and Environmental Studies, University of Yale, USA.
\end{abstract}

\section{RESUMEN}

Un enfoque central de la investigación agrícola en México ha sido la generación de variedades mejoradas de maíz (Zea mays $\mathrm{L}$.) de alto rendimiento y su difusión, pese a que los maíces criollos siguen desempeñando un papel clave en las estrategias de vida de los productores. Existen varias razones, entre ellas las económicas, por las que los productores toman la decisión deliberada de sembrar maíces criollos. El grano de tales variedades puede lograr un sobreprecio si el productor accede a un mercado especializado. Por otra parte, muchas de las variedades criollas generan numerosos productos, más allá del grano, para los que existen mercados importantes, como es el caso de las hojas de maíz ("totomoxtle") que se utilizan para envolver los tamales (platillo tradicional). En el presente documento se examinan con más detalle algunas de las razones económicas que impulsan a los productores a sembrar maíces criollos y se ilustran con ejemplos de los mercados del maíz azul y del maíz para elaborar pozole (platillo tradicional) en la meseta central de México. Para este fin se empleó un enfoque de cadena de valor, que examina las oportunidades y las dificultades a las que se enfrentan los productores al tratar de acceder a estos mercados. La cadena de valor del maíz para pozole depende de la producción local y de las inversiones en infraestructura que contribuyen a actividades que agregan valor, como la producción del pozole precocido. La siembra del maíz azul está más extendida $y$, aunque los productores tienen menos oportunidades de agregarle valor, su grano abastece pequeñas empresas, en su mayoría dirigidas por mujeres. Ambos mercados contribuyen a mejorar las estrategias de vida locales y la conservación in situ de los recursos genéticos. Sin embargo, es necesaria una formulación cuidadosa de las políticas públicas para expandir estos mercados sin disminuir sus beneficios. Esta investigación ayuda a explicar por qué persisten los maíces criollos en la agricultura mexicana, hecho que tiene implicaciones en la dirección que pudiera tomar la investigación agrícola, puesto que brinda a los fitomejoradores y a los nutricionistas información sobre las características agronómicas que los productores necesitan y de la calidad que el mercado exige. Asimismo, la investigación indica que los fitomejoradores, al tiempo que tratan de incrementar la tasa y la estabilidad del rendimiento, también deberían prestar más atención al mejoramiento de las características de calidad valoradas por los productores.

Palabras clave: Zea mays, maíz criollo, mercados, cadena de valor, pozole, maíz azul.

\section{SUMMARY}

A focus of agricultural research efforts in México is on breeding higher-yielding improved maize (Zea mays L.) varieties and enhancing farmer adoption of these varieties despite the fact that maize landraces continue to play a key role in the livelihoods of farmers. There are a number of reasons, not least the economic ones, behind the decision of farmers to grow landraces. Grain from landraces can be more profitable when farmers access specialty maize markets. Furthermore, many landraces produce multiple products, besides grain, for which there is a market, such as husks ("totomoxtle") for wrapping tamales (a traditional dish). We explore in more detail the rationale behind the decision of farmers to grow landraces. For illustrative purposes, we look at maize markets for blue and pozole-making in the Mexican central highlands. Using a value chain approach, we explore the challenges and opportunities farmers face in accessing these markets. The pozole (a traditional dish) value chain relies on localized production and infrastructure investment that contributes to valueadding activities such as producing pre-cooked pozole. Blue maize, meanwhile, is more widely cultivated, and although there are fewer opportunities for farmers to add value, this grain feeds into smallscale, largely female-run businesses. Both markets contribute to local livelihood improvements and in situ conservation, but careful policy design is necessary to scale-up these markets without diminishing their benefits. This research helps explain the persistence of maize landraces in Mexican agriculture. Thus, it has implications for the direction of agricultural research. It provides plant breeders and nutritional experts with information on the agronomic characteristics required by producers and quality traits demanded by the market. It suggests that plant breeders should focus more attention on improving farmeridentified quality characteristics of their landraces along with higher and more stable yields. The desired impact would be a segmented maize seed sector characterized by both improved landraces and improved maize varieties.

Index words: Zea mays, maize landraces, market, added value, pozole, blue maize.

\section{INTRODUCCIÓN}

México es el centro del origen y diversidad del maíz (Zea mays L.). Se han identificado cerca de 56 razas bien definidas (por ejemplo, subpoblaciones que son morfológica y genéticamente distintas), en muchas de las cuales hay un sinnúmero de variedades de los productores (Beadle, 1939; 
Wellhausen et al., 1952; Hernández-Xolocotzi y AlanísFlores, 1970; Ortega y Sánchez, 1989; Piperno y Flannery, 2001; Matsuoka et al., 2002; Ortega, 2003; Lazos y Chauvet, 2011). Es reconocido que los productores conservan la diversidad del cultivo por razones sociales, económicas y culturales, o cuando las variedades locales muestran un comportamiento agronómico superior al de las variedades mejoradas (Bellon y Brush, 1994; Bellon, 1996; Perales et al., 2003; Bellon, 2004; Perales et al., 2005; Latournerie et al., 2006).

En cuanto al comportamiento agronómico, los productores citan con frecuencia la confianza que tienen en los maíces criollos por ser opciones resistentes y predecibles, en comparación con las las variedades "modernas", producto del mejoramiento formal (Arellano y Arriaga-Jordán, 2001). También hay observaciones que apoyan la posibilidad de que las variedades locales sean más confiables que las mejoradas (Bellon et al., 2011; Bellon y Hellin, 2011; Brush, 1995;). En los casos donde se cultivan terrenos marginales, las variedades mejoradas que los científicos identifican como superiores en condiciones experimentales, en realidad pueden rendir menos bajo las condiciones de los productores, debido a las interacciones genotipo $\mathrm{x}$ ambiente que no se manifiestan en los datos recogidos en las parcelas experimentales (Ceccarelli, 1989, 1994; Bellon et al., 2005).

Las variedades criollas locales tienen un papel muy importante en la adaptación al cambio climático en México. En ciertas partes del país es posible que ya exista germoplasma, en forma de las variedades locales, que sería adecuado para sembrar bajo las nuevas condiciones que se predicen como resultado del cambio climático (Bellon et al., 2011; Guarino y Lobell, 2011; Mercer et al., 2012; Ureta et al., 2012). Además, dentro del complejo genético primario del maíz y sus parientes silvestres, existe diversidad genética no aprovechada en forma de caracteres y alelos novedosos (Ortiz et al., 2009), que se podría utilizar para generar nuevas variedades de alto rendimiento y con tolerancia al estrés, mediante la aplicación de los métodos convencionales. Como tal, las variedades criollas locales pueden ser una herramienta importante dentro del arsenal de tecnologías y prácticas que serán necesarias para hacer frente al cambio climático.

Si bien el estudio de las variedades locales de los productores fue desencadenado por la preocupación por su pérdida, en el contexto de la introducción de las variedades mejoradas (Harlan, 1975), los estudios básicos detallados (Brush, 1995; Bellon y Hellin, 2011) han resaltado no solo la vulnerabilidad de las variedades criollas, sino también su gran persistencia. A pesar de los varios decenios de mejoramiento formal y promoción de las variedades resultantes, gran parte de los productores mexicanos sigue sembrando sus variedades locales de maíz (Barkin, 2002). La ineficiencia de las cadenas de semilla explica en parte la no adopción del maíz mejorado, pero también es cierto que los productores toman la decisión deliberada de seguir sembrando las variedades criollas. Existen evidencias de que esto se debe en parte a la existencia de mercados especializados de maíz, que aceptan y a veces exigen características que solo los maíces criollos poseen (Keleman y Hellin, 2009). Este hecho abre las posibilidades de seguir sembrando los maíces criollos, con salida en varios mercados lucrativos, o sea nicho de mercado, aun cuando tengan una aceptación menor en el mercado de grano blanco a gran escala.

Los defensores de la conservación de la agrobiodiversidad han resaltado los posibles vínculos entre las variedades criollas de los agricultores, los mercados agrícolas y las mejores condiciones de vida (Brush, 1991). En todo el mundo hay ejemplos de productores que comercializan sus variedades locales; entre ellas las criollas de frijol (Phaseolus vulgaris L.) en Ecuador (Abbott, 2005), de la papa (Solanum tuberosum L.) en Perú (Devaux et al., 2009), del arroz (Oryza sativa L.) en Tailandia (Brush, 1991, 1995) y de los mijos menores en la India (Gruère et al., 2009). Son ejemplos de la "conservación a través del uso". Sin embargo, el acceso a estos mercados tiene un costo para los productores porque, como sucede en cualquier mercado, la participación en sí implica costos de transacción. Aunque las políticas públicas pueden reducir estos costos para que los agricultores tengan más acceso a los mercados.

Los costos de transacción -que incluyen la búsqueda de información, las negociaciones, la puesta en vigor y el monitoreo de los acuerdos- se generan cuando los bienes se movilizan a lo largo de la cadena de valor al ser producidos, procesados y distribuidos. Una cadena de valor consiste en toda la gama de actividades que se necesita para llevar un producto o servicio desde su concepción, pasando por las distintas etapas de su producción (en las que sucede la transformación física con aportaciones de varios servicios al productor), hasta su entrega a los clientes finales y su eliminación después de su uso (Kaplinsky y Morris, 2000).

En este estudio se abordan algunos aspectos socioeconómicos de la siembra de las variedades de maíz criollo, o sea de los maíces con usos especiales, el acceso a los nichos de mercado (también denominados con el término "mercados especializados" (Hellin y Keleman, 2013)), y las estrategias de vida de los agricultores en México. Este estudio se centra, sobre todo, en los mercados de dos maíces, el azul y el usado para elaborar pozole (platillo tradicional), para ilustrar algunos de los problemas y oportunidades que los productores enfrentan al tratar de acceder a estos mercados especializados, o son mercados fuera del radar 
de los grandes circuitos comerciales. Asimismo, el análisis socioeconómico genera conocimientos acerca de las características agronómicas que los productores requieren y las de calidad que los mercados exigen, que prometen ser de utilidad para los campos técnicos (como el fitomejoramiento y la nutrición) en la investigación científica.

El objetivo de este artículo es demostrar, a través de un estudio del caso de los maíces azul y para pozole en México, que aunque las cadenas de valores son complicadas, los pequeños agricultores tienen acceso a esto nichos de mercado y que los mercados generan beneficios importantes para los mismos agricultores.

Este documento está estructurado de la siguiente manera: primero se describe la metodología utilizada para realizar el estudio. Luego se da una breve introducción al maíz con usos especiales en México, con un enfoque en el maíz azul. Enseguida se enfoca en el Estado de México; se describe la producción y el consumo del maíz en México en general; después se explica la importancia del maíz azul y del maíz para pozole ("pozolero") para los pequeños productores en el Estado de México. En la sección de la discusión se plantean los problemas relacionados con el acceso a los mercados especializados y la reducción de la pobreza. También se describen algunas de las implicaciones para las políticas públicas y la investigación agrícola en el futuro. Para finalizar, se presentan las conclusiones.

\section{METODOLOGÍA}

Gran parte de la investigación descrita en este trabajo fue realizada bajo el rubro de un proyecto sobre los mercados de maíz en el Estado de México. En la metodología abajo descrita se mencionan a todas las personas entrevistadas, aunque los resultados reportados aquí son sobre todo del encuentro con los agricultores, vendedores de maíz y "comaleras" (señoras dedicadas al negocio informal de la venta de antojitos, por ejemplo quesadillas).

La estrategia de mapeo de mercado se basó en Hellin et al. (2005). Una cadena de valor ha sido descrita como "La serie completa de actividades que un producto o servicio requiere desde su concepción, a través de las diferentes fases de producción (que incluyen una combinación de la transformación física y la aportación de varios servicios del productor), envío al cliente final y eliminación última después de usarse" (Kaplinsky y Morris 2000). Una cadena de valor está formada por diversos actores: proveedores de insumos (fertilizantes, semillas y otros), agricultores, comerciantes, procesadores, exportadores/importadores, minoristas y consumidores.

El análisis de la cadena de valor permite efectuar una esti- mación detallada de la manera en que un producto (en este caso, variedades criollas de maíz) se desplaza de un actor a otro a lo largo de la cadena, desde el productor hasta el consumidor. Esta investigación se basa en una combinación de trabajo de campo cualitativo, revisión bibliográfica y análisis de grupos de datos secundarios.

Se puso especial atención tanto en identificar a los actores de la cadena de valor y su relación entre sí como en recabar datos acerca de la rentabilidad relativa de las diferentes variedades de maíz. La revisión bibliográfica se concentró en artículos de publicaciones mexicanas arbitradas y literatura gris de organizaciones estatales y nacionales de apoyo a la agricultura. Las bases de datos con información de la producción agrícola y de las variables socioeconómicas incluyen el Anuario Estadístico del Sistema de Información Agropecuaria (SIAP, 2006), así como del Sistema de Información Geográfica (GIS) e información de población disponible del Instituto Nacional de Estadística, Geografía e Informática (INEGI, 2011).

Después de la exploración inicial de la estructura de la cadena de valor y de la identificación de los actores clave en la cadena de valor, entre octubre de 2007 y junio de 2008 se realizaron entrevistas a representantes de organizaciones gubernamentales, de agricultores, instituciones académicas del Estado de México, organismos públicos de investigación $\mathrm{y}$ fitomejoramiento, pequeños y grandes intermediarios de semillas, compañías productoras de semilla del sector privado, productores de alimento para animales, industria harinera de maíz, sector del nixtamal y productor de tortillas, así como a individuos particulares de los sectores agrícola, productor de alimentos y minorista. Se hizo un total de 67 entrevistas, debido a que en múltiples encuentros se entrevistó a varias personas.

El Cuadro 1 contiene un desglose de los entrevistados por sector. La designación de los individuos como representantes de un sector en particular en ocasiones resulta un tanto artificial, ya que muchos de los entrevistados compartieron su conocimiento acerca de más de una o de varias industrias. Sin embargo, para facilitar la referencia los individuos se clasificaron en categorías de acuerdo con el tema primario que aquí se discute.

Las entrevistas fueron semi-estructuradas con preguntas prediseñadas de acuerdo con la especialización del entrevistado. El contenido y duración de cada una varió según el tiempo y la disposición del entrevistado. Un caso particular fue el de los pequeños agricultores y vendedores privados de grano o de productos de maíz, a quienes se entrevistó al azar en mercados o comunidades. Con el fin de respetar el tiempo de las personas, en general, las entrevistas fueron breves (menos de $15 \mathrm{~min}$ ). Las entrevistas con las personas 
Cuadro 1. Resumen de informantes clave entrevistados por sector/industria (Fuente: Keleman y Hellin, 2009).

\begin{tabular}{lcc}
\hline Tipo de actor en la cadena de valor & $\begin{array}{c}\text { Número de personas } \\
\text { entrevistadas }\end{array}$ & $\%$ del total \\
\hline Agricultores a pequeña escala & 8 & 10.3 \\
Agricultores a escala media/ganadería & 5 & 6.4 \\
Agricultores comerciales/hacendados & 3 & 3.8 \\
Organizaciones de agricultores & 8 & 10.3 \\
Vendedores de maíz en grano & 10 & 12.8 \\
Molinos de granos y propietarios de tortillerías & 7 & 9.0 \\
"Comaleras" & 3 & 3.8 \\
Industria de harina de maíz & 2 & 2.6 \\
Industria de alimentación de ganado & 2 & 2.6 \\
Compañ́as semilleras privadas & 2 & 2.6 \\
Instituciones de investigación pública/fitomejoramiento & 9 & 11.5 \\
Universidades & 2 & 2.6 \\
Dependencias gubernamentales de apoyo agrícola & 7 & 9.0 \\
Vendedores de maíz fresco (elotes/esquites) & 5 & 6.4 \\
Vendedores de hojas de maíz ("totomoxtle”) & 2 & 2.6 \\
Procesadores y vendedores de pozole & 2 & 2.6 \\
Amas de casa no agricultoras & 2 & 1.3 \\
Totales & 78 & 100 \\
\hline
\end{tabular}

a quienes se había contactado con anterioridad o con negocios bien establecidos se hicieron vía telefónica, mediante visitas previamente concertadas o por correo electrónico. Las entrevistas telefónicas y en persona tuvieron una duración de entre 30 min a $2 \mathrm{~h}$.

\section{MAÍCES CON USOS ESPECIALES EN MÉXICO}

En México existen oportunidades de mercado para los maíces criollos. En este contexto, los maíces para especialidades incluyen los maíces de colores (azul, negro, rojo, morado, etc.) y el "pozolero", entre otros. Los consumidores aprecian estos tipos de maíz por sus características culinarias, como el color, la textura, el sabor y su uso en varios platillos típicos. Los productos provenientes de los maíces con usos especiales incluyen también las hojas de mazorca ("totomoxtle") utilizadas en la producción artesanal o para envolver tamales (platillo elaborado con masa de maíz), y el huitlacoche (U. maydis), un hongo que en México se considera un manjar. Estos productos con frecuencia provienen de los maíces criollos porque, en general, se considera que dan productos de mayor calidad (King, 2006; Keleman y Hellin, 2009; Keleman et al., 2013). En la actualidad se ha revivido el interés y el apoyo al sector del maíz en México, y con esto surge la posibilidad de que los productores del maíz criollo puedan incrementar los beneficios de estas oportunidades de mercado.

En México, en 2010, se registró una producción aproximada de 23 millones de toneladas de maíz. Según la base nacional de datos del Sistema de Información Agropecuaria (SIAP, 2006), cerca de $99.5 \%$ de esta producción se comercializó en el mercado en forma de maíz de grano blanco o amarillo para satisfacer las necesidades de la industria de la masa y la tortilla, la industria de harina nixtamalizada, alimentos balanceados y más. El $0.5 \%$ restante fue comercializado como maíz para especialidades, incluyendo los maíces de colores, el "pozolero" y el "palomero" (maíz reventador). En la Figura 1 se muestra que en el caso del maíz con usos especiales, el tipo más importante por volumen de producción es el de colores, y dentro de este grupo el más importante por su cantidad es el azul. En el periodo 2008 - 2010, la proporción de la producción total de granos de maíz de colores se mantuvo en cerca de $0.3 \%$, a pesar de que el valor de este tipo de maíz, en comparación con el valor de la producción total de grano, aumentó ligeramente de $0.30 \%$ en 2008 a $0.35 \%$ en el 2010 (Cuadro 2). Los estados que por lo regular producen maíz de color incluyen Michoacán, Chihuahua, Campeche, México, Tlaxcala, Guerrero y Jalisco. De éstos, los que generan la mayor parte de la producción son Michoacán, Campeche, Chihuahua y México (Cuadro 3). 


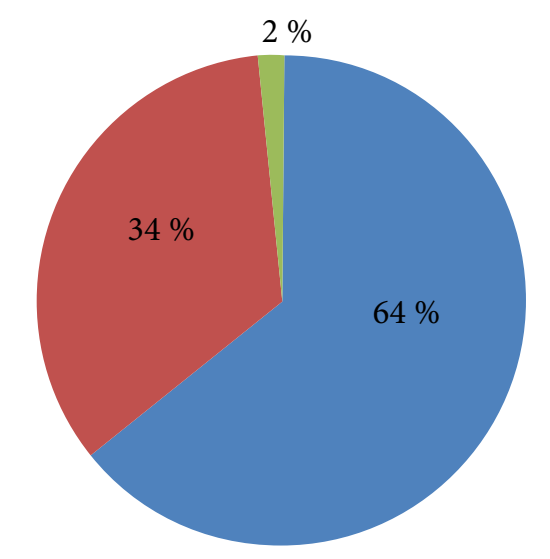

Color "Pozolero" "Palomero"

Figura 1. Distribución de la producción de maíz para especialidades (119 727 t) (Fuente: SIAP, 2010).

Cuadro 2. Producción y valor del total de grano de maíz y la proporción de maíces de colores (Fuente: SIAP, 2010).

\begin{tabular}{lccccc}
\hline \multirow{2}{*}{ Año } & \multicolumn{2}{c}{ Total de grano de maíz } & & \multicolumn{2}{c}{ Maíz de colores } \\
\cline { 2 - 3 } \cline { 5 - 6 } & Producción (millones de t) & Valor (millones de \$) & & Producción (\%) & Valor (\%) \\
\hline 2008 & 24.4 & 68.8 & 56.4 & 0.31 & 0.30 \\
2009 & 20.1 & 65.6 & 0.29 & 0.31 \\
\hline
\end{tabular}

Cuadro 3. Estados de la República Mexicana donde se produce maíz de colores, proporción de la producción y rangos (Fuente: SIAP, 2010).

\begin{tabular}{|c|c|c|c|c|c|c|}
\hline \multirow[b]{2}{*}{ Estados } & \multicolumn{2}{|l|}{2008} & \multicolumn{2}{|l|}{2009} & \multicolumn{2}{|l|}{2010} \\
\hline & $\begin{array}{c}\text { Porcentaje de } \\
\text { producción (\%) }\end{array}$ & Rango & $\begin{array}{c}\text { Porcentaje de } \\
\text { producción (\%) }\end{array}$ & Rango & $\begin{array}{c}\text { Porcentaje de } \\
\text { producción (\%) }\end{array}$ & Rango \\
\hline Campeche & 15.7 & 3 & 11.1 & 3 & 19.7 & 3 \\
\hline Chihuahua & 0.2 & 7 & 5.4 & 6 & 21.8 & 2 \\
\hline Guerrero & 2.6 & 5 & 3.1 & 7 & 2.2 & 6 \\
\hline Jalisco & 2.2 & 6 & 1.7 & 8 & 1.8 & 7 \\
\hline México & 38.3 & 1 & 44.3 & 1 & 16.5 & 4 \\
\hline Michoacán & 32.6 & 2 & 21.0 & 2 & 23.9 & 1 \\
\hline Puebla & - & - & 6.5 & 5 & - & - \\
\hline Querétaro & - & - & - & - & 0.1 & 8 \\
\hline Tlaxcala & 8.5 & 4 & 6.9 & 4 & 13.9 & 5 \\
\hline Producción (t) & 74734 & & 59224 & & 76843 & \\
\hline
\end{tabular}


En 2010, 80 \% de la producción de maíz de colores provino de zonas de temporal (secano) donde el rendimiento promedio es de $1.6 \mathrm{tha}^{-1}$, el $20 \%$ restante se produjo en parcelas irrigadas con un rendimiento promedio de $3.8 \mathrm{t}$ ha $^{-1}$. Según Polanco y Flores (2008), la mayoría del maíz de colores se produce en pequeña escala, en zonas marginadas, y se destina principalmente al consumo doméstico.

El maíz azul tiene un sabor más intenso, más dulce y más especial que las otras variedades sembradas para el consumo humano. Su consistencia granulada produce una tortilla un poco más densa que las elaboradas con harina de maíz blanco o amarillo. En México hay una gran diversidad de variedades de maíz azul que pertenecen a distintas razas; asimismo existe una gran variabilidad en su tamaño, densidad y dureza de grano, y en su composición química (Agama et al., 2011; Salinas-Moreno et al., 2012). En las diferentes regiones productoras de maíz azul se cultivan variedades criollas: en los Valles Altos de la Mesa Central predominan las razas Chalqueño para riego y el Cónico para temporal, aunque también se pueden encontrar Cacahuacintle y Palomero Toluqueño, mientras que en el noroeste la raza que se emplea es Tabloncillo (Salinas et al., 2010; Agama, 2011).

Cabe mencionar que el maíz azul no consiste solo en variedades criollas. En los Estados de Querétaro y Chihuahua hay híbridos como el 'H-512Az' y el 'H-505Az' que se siembran y comercializan bajo la marca Águila. En el Estado de México un criollo mejorado de ICAMEX se comercializa con el nombre de 'Negro Carioca' (Salinas et al., 2010). El INIFAP ha generado las variedades 'V-39 Cocotitlán' para la zona de temporal de Chalco-Amecameca en el Estado de México, 'V-45 Sierra Negra' para la región de SerdánTlachichuca-El Seco en el Estado de Puebla, 'V-43 AZ Malintzi' para los Estados de Tlaxcala e Hidalgo, y 'V-41 AZ Citlali' para Tlaxcala (INIFAP, 2006 y 2009). No obstante, la extensión de la adopción de estas variedades mejoradas puede ser difícil de medir, ya que por lo general se han producido como variedades de polinización abierta en vez de híbridos, y como la producción de semilla no es siempre en cantidades mayores, la difusión es sobre todo a través del sector informal.

En general, el maíz de colores rinde menos que el de grano blanco o amarillo. La mayor diferencia en sus rendimientos se observa en Jalisco, donde el maíz blanco rinde casi seis veces más que el de colores; en Chihuahua, tres veces más; y en Campeche, más del doble. Las diferencias no son tan marcadas en otros estados. El caso de Michoacán es la excepción, ya que allí los maíces de colores rinden más que el de grano blanco (Cuadro 4).

Existen datos distintos de los precios y los sobreprecios que se pagan por el maíz azul. En el ámbito nacional el sobreprecio promedio circunda en $6.7 \%$, pero varía mucho de un estado a otro (Cuadro 5).

\begin{tabular}{lcc}
\multicolumn{3}{l}{$\begin{array}{l}\text { Cuadro 4. Rendimientos de maíz de grano blanco y } \\
\text { maíz de colores }\end{array}$} \\
\hline \multirow{2}{*}{ Estado } & (Fuente: SIAP, 2010). \\
\cline { 2 - 3 } & \multicolumn{2}{c}{ Rendimiento $\left(\mathrm{t} \mathrm{ha}^{-1}\right)$} \\
\hline Campeche & 2.5 & 0.9 \\
Chihuahco & Maíz de color \\
Guerrero & 4.4 & 1.5 \\
Jalisco & 3.0 & 1.9 \\
México & 6.0 & 0.9 \\
Michoacán & 2.9 & 2.8 \\
Querétaro & 3.3 & 3.5 \\
Tlaxcala & 2.7 & 2.4 \\
Total & 2.6 & 2.6 \\
\hline
\end{tabular}

\section{EL MAÍZ EN EL ESTADO DE MÉXICO}

\section{Producción}

Gran parte del Estado de México está entre los 2000 y los $2600 \mathrm{msnm}$. Esta entidad genera cerca de $10 \%$ de la producción nacional de maíz, con dos millones de toneladas al año; su área total de producción de maíz es de unas 600 mil ha (75\% del total de la zona agrícola del estado), con cerca de 230 mil productores que cultivan este grano en una extensión de tierra promedio de 2.5 ha por agricultor, aunque el tamaño de las parcelas fluctúa entre $0.25 \mathrm{y}$ 100 ha, y solo cerca de $3 \%$ supera las 5 ha. En esta zona solo se puede sembrar en un ciclo de maíz por año debido a sus condiciones climáticas, caracterizadas por las bajas temperaturas, heladas y la falta de lluvia desde noviembre hasta abril (Eagles y Lothrop., 1994). Cerca de $15 \%$ de los productores de maíz del estado tienen acceso a riego total o parcial, en tanto que el $85 \%$ restante depende por completo de las lluvias, por lo que siembran después de que éstas comienzan en los meses de mayo y junio.

Los datos estadísticos totales sobre la producción agrícola identifican seis categorías principales de producción de maíz en el Estado de México: (1) forrajero; (2) de grano amarillo; (3) de grano blanco; (4) de grano de colores; (5) "pozolero"; y (6) elotes. Con base en los datos de 2006, cerca de $93 \%$ de la extensión sembrada con maíz (incluye tierras con y sin riego) fue sembrado con maíz de grano blanco (SIAP, 2006). El tipo de maíz que ocupa el segundo lugar en importancia es el forrajero, que cubre cerca de $4 \%$ del total de la extensión sembrada con maíz. El maíz de grano 


\begin{tabular}{|c|c|c|c|c|c|}
\hline \multirow{2}{*}{ Estado } & \multicolumn{3}{|c|}{ Precio medio rural $(\$ / t)$} & \multicolumn{2}{|c|}{ Sobreprecio (\%) } \\
\hline & Blanco & Color & "Pozolero" & Color & "Pozolero" \\
\hline Aguascalientes & 2918 & & 6355 & & 117.8 \\
\hline Campeche & 2934 & 2876 & & -2.0 & \\
\hline Chihuahua & 2485 & 3500 & & 40.8 & \\
\hline Guerrero & 2780 & 3481 & 5968 & 25.2 & 114.7 \\
\hline Jalisco & 2819 & 3720 & & 32.0 & \\
\hline México & 3090 & 3536 & 3200 & 14.4 & 3.6 \\
\hline Michoacán & 2813 & 2525 & & -10.2 & \\
\hline Morelos & 3834 & & 4426 & & 15.4 \\
\hline Puebla & 3393 & & 1483 & & -56.3 \\
\hline Querétaro & 2787 & 2800 & & 0.5 & \\
\hline Tlaxcala & 2810 & 2590 & & -7.8 & \\
\hline Total & 2837 & 3026 & 2861 & 6.7 & 0.8 \\
\hline
\end{tabular}

amarillo, las variedades para especialidades (por ejemplo, el "pozolero" y las de colores) y las de elotes representaron cada uno $1.5 \%$ de toda el área cosechada, aproximadamente. Las variedades de maíz criollo cubren más de $80 \%$ del área sembrada con maíz en esta entidad. Las cifras del SIAP sugieren que cada año se producen poco menos de 50 mil t de grano de variedades con usos especiales, que incluyen a los maíces de colores (rojo, rosa o azul) y al "pozolero" (Cuadro 6).

\section{Consumo}

El maíz producido en el Estado de México no es suficiente para satisfacer la demanda de toda la entidad. El consumo total es de 3.6 millones de toneladas al año y, por ende, hay una diferencia de $40 \%$ entre la producción y el consumo. Por esta razón se importa maíz de otros estados de la República Mexicana y de los Estados Unidos. Casi 50 \% del maíz que se consume en el Estado de México se destina al uso doméstico o se vende en mercados diferenciados (con frecuencia, informales) de maíz (Cuadro 7). El siguiente nivel más alto de consumo consiste en la industria de harina de maíz procesada, de la masa-tortilla y la del almidón de maíz industrializado. El quinto lugar es el maíz para la alimentación animal.

Si bien existe una gran demanda de mercado de maíz de grano blanco y harinoso para hacer tortillas, los productores informan que tienen dificultad para participar en los mercados de maíz a grano porque las variedades criollas que siembran no poseen algunas de las características de calidad que las agroindustrias procesadoras requieren, como la forma del grano (el grano de maíz de estos produc- tores es redondo y puntiagudo, y no del tipo plano que las procesadoras exigen) y el color del grano (es cremoso, no blanco como las procesadoras exigen). En cuanto al acceso al mercado, el maíz criollo es mucho más aceptado por las pequeñas empresas, a menudo del sector informal, que son conocidas de manera informal como "comaleras", cuyos productos primarios son los antojitos elaborados con maíz, como las quesadillas, los tlacoyos y los tacos.

\section{Productos de maíz con usos especiales}

El maíz para especialidades suele recibir un sobreprecio en comparación con el vendido en los mercados a granel, además algunos mercados especializados son más accesibles para los agricultores que producen volúmenes pequeños del grano. Los datos del SIAP sugieren que el Estado de México es el productor más importante a nivel nacional de variedades de maíz para especialidades. En 2006, por ejemplo, la producción de maíz "pozolero" en la entidad representó $54.5 \%$ del total nacional registrado, en tanto que la producción de maíces de colores resultó en $34.4 \%$ del total nacional (SIAP, 2006).

En el Estado de México existen mercados activos especializados para:

- Maíz de color azul, ingrediente con que se elaboran los antojitos.

- Maíz harinoso de grano grande para hacer pozole.

- Totomoxtle (hojas de la mazorca) para envolver tamales.

- Maíz rojo o rosa para alimentar a los animales y, a 
Cuadro 6. Producción de grano de maíz en el Estado de México (Fuente: SIAP 2005 y 2006).

\begin{tabular}{lcc}
\hline & \multicolumn{2}{c}{2006} \\
\cline { 2 - 3 } & $\begin{array}{c}\text { Producción de maíz (miles } \\
\text { de toneladas) }\end{array}$ & $\begin{array}{c}\text { Proporción del total en } \\
\text { el estado (\%) }\end{array}$ \\
\hline Amarillo & 12.3 & 0.7 \\
Blanco & 1740.5 & 96.6 \\
De colores & 23.3 & 1.3 \\
"Pozolero" & 25.3 & 1.4 \\
Total de grano de maíz & 1801.4 & \\
\hline
\end{tabular}

Cuadro 7. El consumo de maíz en el Estado de México (2008) (Fuente: Keleman y Hellin, 2009).

\begin{tabular}{|c|c|c|}
\hline Consumidor & $\begin{array}{l}\text { Consumo anual } \\
\text { (miles de } \mathrm{t})\end{array}$ & $\begin{array}{l}\text { Consumo anual en } \\
\text { el estado (\%) }\end{array}$ \\
\hline Industria de harina de maíz procesada & 500 & 13.9 \\
\hline Industria de harina molida y de la tortilla & 525 & 14.6 \\
\hline Producción de animales & 383 & 10.6 \\
\hline Aves & 232 & 6.4 \\
\hline Porcinos & 31 & 0.9 \\
\hline Bovinos y lácteos & 120 & 3.3 \\
\hline Almidón de maíz industrializado & 500 & 13.9 \\
\hline $\begin{array}{l}\text { Consumo de subsistencia y mercados } \\
\text { diferenciados de maíz }\end{array}$ & 1700 & 47.1 \\
\hline Total & 3608 & \\
\hline
\end{tabular}

veces, para hacer tortillas.

- Elotes asados, hervidos o desgranados para vender.

Estos mercados especializados comparten algunas características en común, tanto en infraestructura como en organización. En primer lugar tienen una baja concentración; a pesar de que hay algunos compradores industriales importantes, éstos representan una participación mínima del mercado y reflejan las preferencias específicas del consumidor. Por ejemplo, los compradores urbanos valoran la frescura en los antojitos del maíz "pozolero" y del azul, pero tienden a confiar en el sector informal (es decir, puestos en la vía pública) en cuanto a productos de alta calidad se refiere, en lugar de buscarlos en restaurantes o prepararlos en casa.

A pesar de que existe una baja concentración económica en estos mercados, geográficamente se localizan en los mercados municipales de la región. La Central de Abastos en la ciudad de México (un mercado de mayoreo) es el mejor prototipo y el más influyente de éstos, ya que abastece de productos agrícolas y secos a la mayoría de los mercados locales de la ciudad, incluso a muchos supermercados.
En la Figura 2 se muestra un esquema de la cadena de valor del maíz azul. En febrero de 2008 los agricultores que vendían maíz azul a las bodegas locales, que fungen como intermediarias, recibieron un precio entre 2.3 y 2.5 pesos/ $\mathrm{kg}$ ( $\$ 0.22$ dólares estadounidenses, Cuadro 8), mientras que los agricultores que vendieron el maíz en los mercados locales ("tianguis") pudieron obtener hasta 4 pesos $/ \mathrm{kg}$ ( $\$ 0.38$ dólares estadounidenses). Otra estrategia es la venta directa del maíz a las "comaleras"; un agricultor mediano de Acambay, que produce unas $20 \mathrm{t}$ de maíz azul al año, reportó una distribución de hasta $1 \mathrm{t}$ cada dos semanas a varias mujeres con negocios de antojitos. El grano se vendió a 4 pesos $/ \mathrm{kg}$ ( $\$ 0.38$ dólares estadounidenses).

Antonio et al. (2004) estimaron que una tercera parte de los productores de maíz de temporal en el estado siembran cerca de una tercera parte de sus tierras con maíz azul. Sin embargo, existe poca información de la cantidad que se siembra en las fincas. Los productores aquí entrevistados dijeron sembrar distintas extensiones con maíz azul, desde una parcela de $300 \mathrm{~m}^{2}$ hasta 5 ha. Ninguno de los grandes productores (es decir, mayores a $30 \mathrm{ha}$ ) informó que siembre grandes extensiones con maíz azul. 


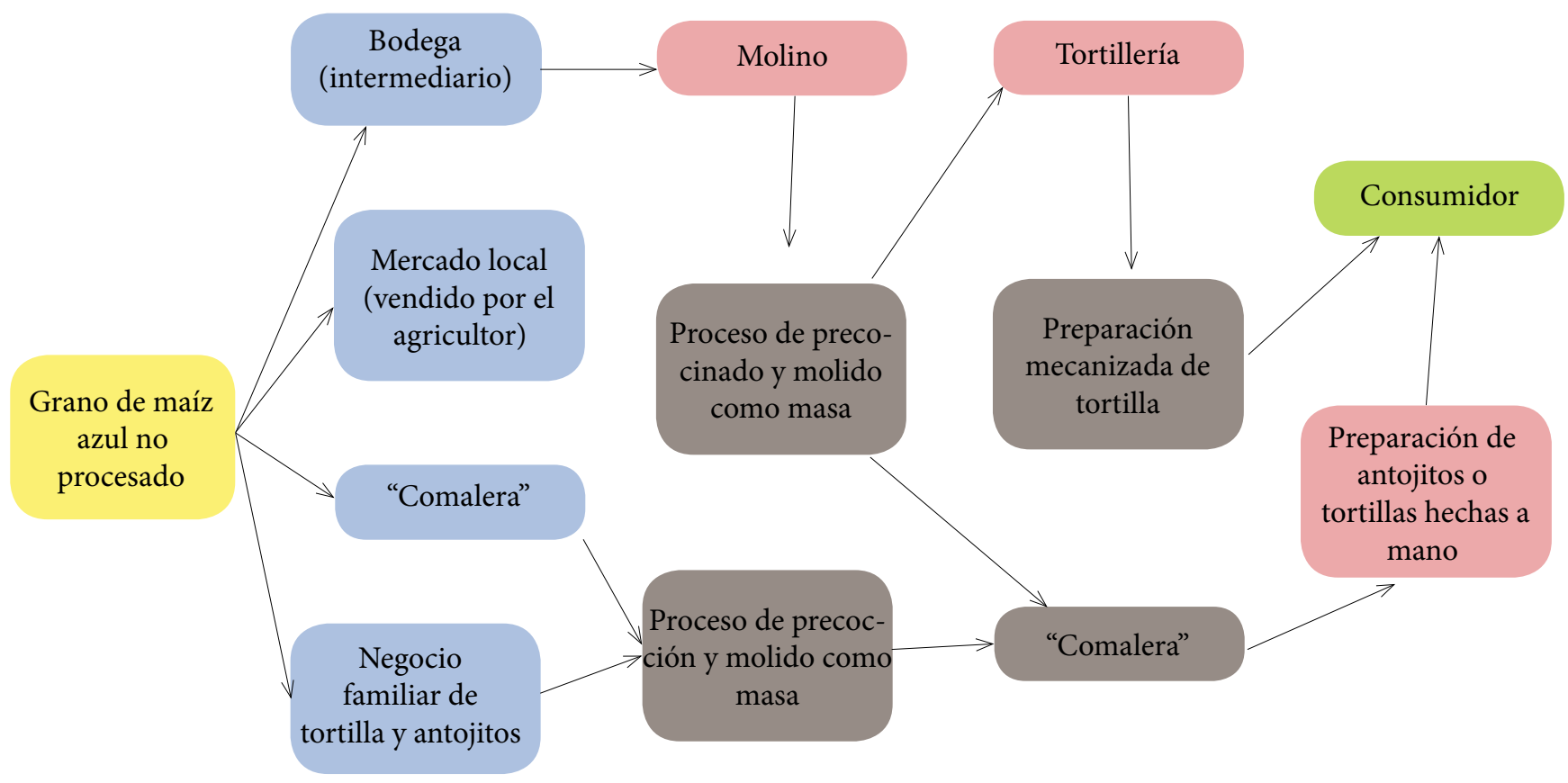

Figura 2. Cadenas de valor para el grano de maíz azul. Los puntos de procesamiento están al centro, en color gris (Fuente: trabajo de campo de los autores).

El grano de maíz azul recibe un sobreprecio aproximado de 10 a $15 \%$ en comparación con el maíz blanco. Los productores que se saltan a los intermediarios y venden de manera directa a las procesadoras reciben un sobreprecio todavía mayor (Figura 2). Uno de los aspectos clave que convence a los productores a utilizar las estrategias que eliminan a los intermediarios, son las empresas informales que venden antojitos, como quesadillas o tortillas hechas a mano. Aunque es posible encontrar productos elaborados con maíz azul en los restaurantes, el consumidor urbano en México acostumbra comer estos productos en puestos localizados en las calles o en las plazas regionales. En las zonas urbanas los antojitos se venden a entre 9 y 12 pesos (equivalente a 0.86 a 1.14 dólares estadounidenses) la pieza (por ejemplo, una quesadilla) y las tortillas de maíz azul hechas a mano se venden hasta en 13 ó 15 pesos la docena (equivalente a 1.24 dólares por $3 / 4$ de kilogramo de tortillas, aproximadamente). En el caso de las tortillas hechas de maíz azul, este precio fluctúa entre 104 y $135 \%$ más que el de las tortillas de maíz blanco elaboradas en máquina. Pese a que la producción de antojitos implica mayores costos de mano de obra y de insumos, incluso así genera un buen ingreso para las familias y las empresas operadas por mujeres.

Dos tipos de maíz dan un pozole de gran calidad: el Ancho y el Cacahuacintle; algunos productores siembran el maíz Palomo (de la raza Chalqueño) que también sirve para hacer pozole. Aquí el artículo se centra en primer lugar en el Cacahuacintle, ya que el Palomo resulta en un pozole de calidad inferior y es más susceptible al daño por insectos durante su almacenamiento. Los productores suelen sembrar maíz Ancho a una altitud menor de $2000 \mathrm{~m}$ y, por tanto, es más común en el vecino Estado de Morelos. El Cacahuacintle es una raza de maíz cuyo grano es harinoso, grande y blanco (Vazquez et al., 2011). Algunos informadores importantes y la base de datos del SIAP (2006) indican que la producción de maíz "pozolero" de calidad está muy localizada en el Estado de México; el principal centro de producción de este maíz es el municipio de Calimaya, cerca de Toluca, aunque también se produce en la parte sudoriental del estado, en particular cerca del municipio de Chalco.

El Cacahuacintle se vende en forma de grano no procesado (sin valor agregado), descabezado o pozole precocido (Bonifacio et al., 2005). Los granos de Cacahuacintle, que son de gran calidad, se venden entre 5 y 6 pesos el kilogramo (equivalente a entre 0.48 y 0.57 dólares) y cuando hay escasez (como sucedió en 2007) el precio puede alcanzar los 12 pesos por kilogramo (equivalente a 1.14 dólares). Estos precios representan un sobreprecio sustancial en comparación con el valor al productor del maíz blanco no procesado que se situó en 2.10 pesos por kilogramo (0.20 dólares) en febrero de 2008. El maíz "pozolero" de baja 
Cuadro 8. Precios y primas de precios para productos de granos de maíz, en pesos mexicanos (Fuente: Keleman y Hellin, 2009).

\begin{tabular}{|c|c|c|c|c|}
\hline Producto & Tipo & Mercado & $\begin{array}{l}\text { Precio de mercado } \\
\text { (precio/kilo) }\end{array}$ & $\begin{array}{c}\text { Margen bruto sobre } \\
\text { grano de maíz blanco (\%) }\end{array}$ \\
\hline \multicolumn{5}{|l|}{ Grano } \\
\hline \multirow{2}{*}{$\begin{array}{l}\text { Maíz blanco para } \\
\text { harina }\end{array}$} & & $\begin{array}{l}\text { Mercado de productos } \\
\text { básicos a granel, vía } \\
\text { intermediario }\end{array}$ & 21 & $(\mathrm{n} / \mathrm{d})$ \\
\hline & $\begin{array}{c}\text { No procesado: calidad } \\
\text { promedio }\end{array}$ & $\begin{array}{l}\text { Mercado local directo al } \\
\text { consumidor }\end{array}$ & 3.3 & 58.6 \\
\hline \multirow{3}{*}{ Maíz “pozolero” } & $\begin{array}{l}\text { No procesado: calidad } \\
\text { baja }\end{array}$ & Vía intermediario & $\begin{array}{l}3-4 \\
5-6\end{array}$ & $\begin{array}{c}42.8-90.5 \\
138.1-185.7\end{array}$ \\
\hline & $\begin{array}{l}\text { No procesado: calidad } \\
\text { alta }\end{array}$ & $\begin{array}{l}\text { Vía intermediario con } \\
\text { desabasto del mercado }\end{array}$ & 12 & 471.4 \\
\hline & & $\begin{array}{l}\text { Vendido a la cooperativa } \\
\text { "Pozole el Centenario" }\end{array}$ & 5 & 138.1 \\
\hline \multirow{2}{*}{ Maíz azul } & $\begin{array}{l}\text { No procesado: calidad } \\
\text { promedio }\end{array}$ & Bodega local (intermediario) & $23-25$ & $9.5-19.0$ \\
\hline & $\begin{array}{c}\text { No procesado: calidad } \\
\text { promedio }\end{array}$ & $\begin{array}{l}\text { Mercado local, directo al } \\
\text { consumidor }\end{array}$ & 4 & 90.5 \\
\hline \multicolumn{5}{|c|}{ Grano de valor agregado } \\
\hline \multirow{3}{*}{ Maíz “pozolero” } & $\begin{array}{l}\text { Pozole empacado: maíz } \\
\text { precocido }\end{array}$ & Mayoristas, minoristas & $12-20^{\dagger}$ & $471.4-852.4$ \\
\hline & \multirow{2}{*}{ Descabezado } & Vía intermediario & $4.5^{\dagger \dagger}$ & 114 \\
\hline & & Mercado al por mayor & $7^{\dagger \dagger}$ & 233 \\
\hline Tortilla & & & & $\begin{array}{l}\text { Margen bruto sobre la } \\
\text { tortilla de maíz blanco }\end{array}$ \\
\hline $\begin{array}{l}\text { Tortilla de maíz } \\
\text { blanco }\end{array}$ & $\begin{array}{l}\text { Producción masiva a } \\
\text { partir de harina preem- } \\
\text { pacada }\end{array}$ & $\begin{array}{l}\text { Al menudeo (venta directa al } \\
\text { consumidor) }\end{array}$ & 8.5 & $(\mathrm{n} / \mathrm{d})$ \\
\hline $\begin{array}{l}\text { Tortilla de maíz } \\
\text { azul }\end{array}$ & $\begin{array}{l}\text { Hecha a mano de masa } \\
\text { fresca }\end{array}$ & $\begin{array}{l}\text { Al menudeo (venta directa al } \\
\text { consumidor) }\end{array}$ & $17.3-20$ & $103.5-135.3$ \\
\hline
\end{tabular}

Fuente: trabajo de campo; Nota: el tipo de cambio en junio de 2008 era de $\$ 10.5$ MXN por dólar estadounidense.

${ }^{\dagger}$ Este precio incluye el peso del agua, por lo que es probable que se subestime el valor total por kilo de grano crudo.

${ }^{\dagger+}$ Este precio excluye el peso de la proporción del grano que ha sido removido en el proceso del valor agregado, por lo que es probable que se sobreestime el valor total por kilo de grano crudo.

calidad suele procesarse para producir maíz descabezado, donde se retira el grano de la mazorca mediante un aparato que parece un serrucho pequeño. A los granos de menor tamaño y de poca calidad se les corta la punta para facilitar la absorción de agua durante su cocción; el grano descabezado es procesado a mano por el (la) productor (a) y su familia, o en industrias caseras locales; tiene un precio de alrededor de 4.5 pesos por kilogramo y se puede vender a través de un intermediario o directamente al consumidor en los mercados locales.

El mayor valor es agregado al preparar pozole precocido. Este proceso dura dos días; se hierve el grano en hidróxido de sodio o de potasio y luego se deja remojar toda una noche. El grano cocido se prepara para almacenarse y venderse, a veces en bolsas de plástico selladas o se guarda en cubetas. Un kilogramo de este grano se vende entre 12 y 20 pesos (entre 1.20 y 1.90 dólares). El proceso de cocción del Cacahuacintle para hacer pozole es largo y muchos consumidores urbanos prefieren comprarlo ya precocido. Se puede hacer un guiso con este maíz precocido, después de hervirlo durante unas cuantas horas, lo cual reduce la mano de obra doméstica contratada (o no) que se requiere para elaborar el producto final (Bonifacio et al., 2005). Estas oportunidades lucrativas que agregan valor han contribuido al crecimiento de muchas industrias caseras familiares y cooperativas de agricultores. Algunas de estas entidades productoras han establecido maneras de marcar y etiquetar 
sus productos con el nombre del maíz criollo utilizado y con su región de origen, como indicadores de su calidad (Keleman y Hellin, 2009).

El sobreprecio no es una razón principal que explique la siembra del maíz criollo, sino que es importante saber cuál es el ingreso por hectárea (precio/rendimiento) y los costos de producción. Los ingresos brutos y netos de los agricultores en este estudio han sido reportado por Keleman et al. (2013), que si bien estos autores no distinguieron entre las diferentes variedades criollas y no incluyeron variedades mejoradas de maíz, ellos demostraron que los beneficios a los agricultores son mucho mayores por la comercialización directa del grano en los mercados de especialidades en comparación con su venta a un intermediario, porque así pueden aumentar sus ingresos en casi $50 \%$.

\section{DISCUSIÓN}

\section{Maíces con usos especiales y reducción de la pobreza}

Un dato clave que la investigación sobre el maíz con usos especiales en México ha revelado es la función importante que las variedades de este maíz tienen en los ingresos y las oportunidades de mercado de los productores. El maíz con usos especiales representa un pequeño porcentaje de toda la producción del maíz nacional, pero su producción tiende a efectuarse por agricultores de escasos recursos (Antonio et al., 2004). Esto significa que aunque solo se lograra dar a los productores pobres un acceso mínimo a los mercados especializados más lucrativos, esto probablemente tendría un impacto importante en la reducción de la pobreza. No obstante, los pequeños productores de los maíces azules y "pozoleros" a menudo enfrentan problemas relacionados con altos costos de transacción por unidad al obtener acceso a capital, información de mercado y técnica, mercados de mercancías y producción, y proporcionar rastreabilidad y control de calidad al producto.

Los pequeños y medianos agricultores que generan muchos productos de maíz para después venderlos en los mercados especializados, reportan un beneficio neto por hectárea que equivale al doble del que obtienen los productores que venden el grano en los mercados de productos comerciales (Keleman y Hellin, 2009). Sin embargo, debido al volumen de su producción los grandes agricultores generan mayores ingresos aunque cosechen un número menor de productos a través de técnicas mecanizadas y mano de obra contratada, para después venderlos en los mercados industriales. Estas observaciones ayudan a entender el por qué en vez de sembrar híbridos de alto rendimiento, los pequeños productores siguen cultivando maíz criollo para venderlo en los mercados especializados, y también porqué los productores no han adoptado ampliamente el germoplasma mejorado que genera mayores rendimientos de grano.

La razón principal para que los productores de maíces tengan acceso a los mercados especializados, es el bajo nivel de concentración en las cadenas de valores. Aunque existen algunos grandes compradores industriales, éstos tienden a una participación mínima en el mercado, lo cual refleja las preferencias específicas de los consumidores: por ejemplo, a los urbanos les gusta que el pozole y los antojitos elaborados con maíz azul estén recién hechos y prefieren consumir los de alta calidad en el sector informal (es decir, en un puesto en la calle), en lugar de ir a un restaurante formal o prepararlos en casa. No obstante, también existen diferencias: en el caso del maíz "pozolero" los productores le añaden gran parte de su valor agregado; pero los productores más pobres no suelen tener acceso a la infraestructura ni a la tecnología necesarias para agregar valor. Por tanto, si bien es cierto que este mercado tiene efectos localizados importantes en los ingresos de los agricultores, sería muy difícil tratar de aumentarlos con miras a reducir más la pobreza.

Por el contrario, en el mercado del maíz azul las pequeñas empresas que están vinculadas de manera estrecha con las comunidades agrícolas son las que le agregan casi todo el valor al producto. En muchos casos, los agricultores no reciben los beneficios por la venta del producto final al consumidor, pero sí se favorecen con los sistemas que eliminan a los intermediarios. Pese a esta desventaja, el potencial en pro de los pobres del mercado del maíz azul es mayor que el del mercado del maíz "pozolero", ya que existen menos barreras que impiden a los productores acceder al primero. Según la gente entrevistada, la cadena de valor del maíz azul también beneficia a las pequeñas empresas que suelen ser operadas por mujeres.

\section{Políticas públicas y la estimulación de los nichos de mercado}

El maíz azul y el maíz "pozolero" revelan un importante reto de los mercados especializados, es decir, que si bien éstos tienen el potencial de contribuir a reducir la pobreza, es probable que no se realice si pequeños grupos de productores y procesadores relativamente más ricos reciben la mayor parte de las utilidades generadas. En la actualidad, el gobierno y las organizaciones no gubernamentales (ONG) intervienen muy poco en las cadenas de valor y, por tanto, éstas son coordinadas sobre todo por medio de las iniciativas del sector privado, cooperativas de productores y relaciones comerciales basadas en nexos sociales.

En el contexto de cualquier intento del gobierno por estimular el crecimiento de los nichos de mercado, un aspecto importante sería elaborar estrategias que promuevan la 
equidad en las cadenas de valor sin inhibir ni distorsionar la función de los mercados especializados, además de no obstaculizar la función crucial del sector privado. Las estrategias dirigidas al incremento de los efectos a favor de los pobres en el mercado del maíz azul y del "pozolero", deberían promover los micropréstamos ofrecidos por proveedores privados y públicos, así como los servicios de desarrollo empresarial, con el fin de fomentar el crecimiento de la pequeña industria ya existente y que agrega valor.

La función que los intermediarios externos y de las organizaciones sociales podrían desempeñar en fomentar los mercados especializados, es un tema que merece ser investigado. En el mercado del maíz "pozolero" durante el proceso de la identificación de los informadores clave para ser entrevistados, se encontró que han surgido líderes entre los productores de este maíz. En algunos casos estos contactos clave estaban en posición de facilitar la diseminación de los conocimientos provenientes de las fuentes externas a sus comunidades, y ya habían sido identificados por los institutos locales de desarrollo rural con este mismo propósito. No obstante, la función de estos individuos clave en general se había definido por su pertenencia a un grupo social dado (por ejemplo, a una asociación comercial o familiar), por lo cual no quedaba claro hasta qué punto estarían dispuestos a compartir sus conocimientos con un público más amplio, es decir, con otros productores del maíz "pozolero".

La función potencial de los intermediarios externos es menos transparente en el sistema de maíz azul, debido a que la organización social que rodea a la producción de este maíz es más débil y no ha sido definida por las mismas limitaciones geográficas que tiene el maíz "pozolero". En teoría, podrían surgir organizaciones de productores que se dediquen a la producción del maíz azul (por ejemplo, con el fin de promover mejores precios para el maíz azul en general), pero éstas enfrentarían los mismos problemas que encaran las asociaciones de productores en todo el mundo y que han sido documentados en las redes de maíz de México (Hellin et al., 2009).

\section{Implicaciones para los fitomejoradores}

Los fitomejoradores han dedicado poca atención a las variedades de maíz criollo o a los aspectos de la calidad de su grano; por lo regular sus esfuerzos se han enfocado en el aumento del rendimiento. Los resultados de la investigación sobre los mercados de maíz para especialidades señalan que existe potencial para enfocar el mejoramiento del maíz, sobre todo en las características de calidad de los maíces criollos. Al reconocer la importancia que las características de la calidad tienen en los mercados de tortilla, harina de maíz y de maíz para especialidades, se llega a la conclusión de que el enfoque del fitomejoramiento debe ampliarse, es decir, que no debe centrarse solo en optimizar el rendimiento del grano y su tolerancia al estrés, sino que tendría que incluir también la gama de características agronómicas y de calidad para las cuales existe una demanda de mercado. En conclusión, es probable que este tipo de investigación dé como resultado estrategias de vida más seguras para un mayor número de productores que hasta ahora, porque no se han beneficiado de la investigación agrícola.

\section{CONCLUSIONES}

Si bien las deficiencias de las cadenas que proveen semillas explican en parte la falta de adopción del maíz mejorado, también es cierto que los productores toman la decisión deliberada de no adoptar las variedades mejoradas. Por otro lado, las variedades criollas de maíz tienen una función importante en los ingresos y en las oportunidades de los productores mexicanos. Los ejemplos aquí descritos de las cadenas de valor del maíz para elaborar pozole y maíz azul en el Estado de México, demuestran que en los mercados de estos maíces en dicha entidad se han establecido vínculos entre los distintos actores de la cadena desde hace muchos años. Estas cadenas de valor permiten a los productores generar mayores ingresos y conservar in situ las variedades locales de maíz criollo.

Esto tiene consecuencias de gran alcance para las políticas agrarias de México. Este artículo se ha enfocado en el maíz azul y en el "pozolero", pero hay otros nichos de mercado para otros productos, como el "totomoxle". En lugar de medir la eficiencia de los productores solo con base en su producción de grano, una mejor estrategia sería reconocer al maíz como un cultivo que genera una gran cantidad de productos, algunos de los cuales se venden en muchos mercados, lo que daría un mejor respaldo a las estrategias agrarias y de sustento que los productores ya practican con el fin de aumentar al máximo sus utilidades por hectárea. Las políticas que refuerzan una diversidad de estrategias de cultivo de maíz tendrían un efecto positivo en el desarrollo rural y en la preservación de la agrobiodiversidad en la finca.

\section{AGRADECIMIENTO}

Al Departamento de Agricultura de Estados Unidos (USDA, por sus siglas en inglés) y a la Secretaría de Agricultura, Ganadería, Desarrollo Rural, Pesca y Alimentación (SAGARPA) de México, que financiaron el análisis original. Este documento se basa en la investigación realizada por el proyecto de investigación de USDA y SAGARPA, actualiza el material y expande la revisión bibliográfica. La publicación de este manuscrito ha sido apoyada por "MasAgro: Modernización Sustentable de la Agricultura Tradicional", 
un programa de SAGARPA-CIMMYT. A N. Palacios Rojas, Begoña Bolaños Meade y al Comité Editorial de la Revista Fitotecnia Mexicana, cuyas revisiones editoriales permitieron mejorar la versión original.

\section{BIBLIOGRAFÍA}

Abbott J A (2005) Counting beans: agrobiodiversity, indigeneity, and agrarian reform. The Prof. Geographer 57:198-212.

Agama A E, Y Salinas M, G Pacheco V, L A Bello P (2011) Características físicas y químicas de dos razas de maíz azul: morfología del almidón. Rev. Mex. Cienc. Agríc. 2:317-329.

Antonio Miguel M, J L Arellano Vázquez, G García de los Santos, S Miranda Colín, J Apolinar Mejía Contreras, F V González Cossío (2004) Variedades criollas de maíz azul raza Chalqueño: características agronómicas y calidad de semilla. Rev. Fitotec. Mex. 27:9-15.

Arellano Hernández A, C Arriaga-Jordán (2001) Why improved maize (Zea mays) varieties are utopias in the highlands of central Mexico. Convergencia 8:255-276.

Barkin D (2002) The reconstruction of a modern Mexican peasantry. J. Peasant Stud. 30:73-90.

Beadle G W (1939) Teosinte and the origin of maize. J. Hered. 30:245-247.

Bellon M R (1996) The dynamics of crop infraspecific diversity: A conceptual framework at the farmer level. Econ. Bot. 50:26-39.

Bellon M R (2004) Conceptualizing interventions to support on-farm genetic resource conservation. World Develop. 32:159-172.

Bellon M R, S B Brush (1994) Keepers of maize in Chiapas, Mexico. Econ. Bot. 48:196-209

Bellon M R, J Hellin (2011) Planting hybrids, keeping landraces: agricultural modernization and tradition among small-scale maize farmers in Chiapas, Mexico. World Develop. 39:1434-1443. Doi:10.1016/j.worlddev.2010.12.010.

Bellon M, D Hodson, D Bergvinson, D Beck, E Martínez Romero, Y Montoya (2005) Targeting agricultural research to benefit poor farmers: Relating poverty mapping to maize environments in Mexico. Food Policy 30:476-492.

Bellon M R, D Hodson, J Hellin (2011) Assessing the vulnerability of traditional maize seed systems in Mexico to climate change. Proc. Natl. Acad. Sci. USA. 108:13432-7. doi:10.1073/ pnas.1103373108.

Bonifacio V E I, Y Salinas M, A Ramos R, A Carrillo O (2005) Calidad pozolera en colectas de maíz cacahuacintle. Rev. Fitotec. Mex. 28:253-260.

Brush S B (1991) A farmer-based approach to conserving crop germplasm. Econ. Bot. 45(2): 153-165.

Brush S B (1995) In situ conservation of landraces in centers of crop diversity. Crop Sci. 35:346-354.

Cecarelli S (1989) Wide adaptation: How wide? Euphytica 40:197-205.

Cecarelli S (1994) Specific adaptation and breeding for marginal conditions. Euphytica 77:205-219.

Devaux A, D Horton, C Velasco, G Thiele, G López, T Bernet, I Reinoso, M Ordinola (2009) Collective action for market chain innovation in the Andes. Food Policy 34:31-38.

Eagles H A, J E Lothrop (1994) Highland maize from Central Mexico- its origin, characteristics, and use in breeding programs. Crop Sci. 34:11-19.

Gruère G, L Nagarajan, E D I O King (2009) The role of collective action in the marketing of underutilized plant species: Lessons from a case study on minor millets in South India. Food Policy 34:39-45.

Guarino L, D B Lobell (2011) A walk on the wild side. Nature Clim. Change 1;374-375. doi:10.1038/nclimate1272.

Harlan J (1975) Our vanishing genetic resources. Science 188:618-621.

Hellin J, A Griffith, M Albu (2005) Mapping the market: market-literacy for agricultural research and policy to tackle rural poverty in Africa. In: Beyond Agriculture - Making Markets Work for the Poor. F R Almond, S D Hainsworth (eds). pp: 109-148. Proc. International Seminar, 28 February-1 March, 2005. Westminster, London, UK. Crop Post-Harvest Programme (CPHP), Natural Resources International Limited, Aylesford, Kent and
Practical Action, Bourton-on-Dunsmore, Warwickshire, UK.

Hellin, J, A Keleman (2013) Las variedades criollas del maíz, los mercados especializados y las estrategias de vida de los productores. LEISA Magazine 29:7-9.

Hellin J, M Lundy, M Meijer (2009) Farmer organization, collective action and market access in Meso-America. Food Policy 34:1622.

Hernández-Xolocotzi E, G Alanís-Flores (1970) Estudio morfológico de cinco nuevas razas de maíz de la Sierra Madre Occidental de México. Agrociencia 5:3-30.

INEGI, Instituto Nacional de Geografía y Estadística (2011) Población: Rural y urbana. Disponible en: http://cuentame.inegi.org.mx/ poblacion/rur_urb.aspx?tema=P (Agosto 2012).

INIFAP, Instituto Nacional de Investigaciones Forestales, Agrícolas y Pecuarias (2006) Innovaciones Tecnológicas 2005. Para Mejorar la Competitividad y Sostenibilidad de las Cadenas Agroalimentarias y Agroindustriales. Folleto Técnico 4. Instituto Nacional de Investigaciones Forestales, Agrícolas y Pecuarias, México D.F. 120 p.

INIFAP, Instituto Nacional de Investigaciones Forestales, Agrícolas y Pecuarias (2009) Informe Anual de Actividades 2008. Instituto Nacional de Investigaciones Forestales, Agrícolas y Pecuarias, México D.F. 60 p.

Kaplinsky R, M Morris (2000) A Handbook for Value Chain Research. International Development Research Center (IDRC), Canada. $113 \mathrm{p}$.

Keleman A, J Hellin (2009) Specialty maize varieties in Mexico: A case study in market-driven agro-biodiversity conservation. J. Latin Amer. Geogr. 8:147-174.

Keleman A, J Hellin, D Flores (2013) Diverse varieties and diverse markets: scale-related maize "profitability crossover" in the central Mexican Highlands. Hum. Ecol. DOI 10.1007/s10745-0139566-z.

King A (2006) Trade and totomoxtle: Livelihood strategies in the Tontonacan region of Veracruz, Mexico. Agric. Human Values 24:29-40.

Latournerie Moreno L, J Tuxill, E Yupit Moo, L Arias Reyes, J C Alejo, D I Jarvis (2006) Traditional maize storage methods of mayan farmers in Yucatan, Mexico: Implications for seed selection and crop diversity. Biodivers. Conserv. 15:1771-1795.

Lazos E, M Chauvet (2011) Análisis del contexto social y biocultural de las colectas de maíces nativos en México. Proyecto Global de Maíces, Informe de Gestión, CONABIO. Disponible en: http:// www.bergfiles.com/i/bf4a076dfbh32i0.

Matsuoka Y, Y Vigouroux, M M Goodman, J Sanchez G, E Buckler, J. Doebley (2002) A single domestication for maize shown by multilocus microsatellite genotyping. Proc. Natl. Acad. Sci. USA 99:6080-6084.

Mercer K L, H R Perales, J D Wainwright (2012) Climate change and the transgenic adaptation strategy: Smallholder livelihoods, climate justice, and maize landraces in Mexico. Global Environ. Change 22:495-504. doi:10.1016/j.gloenvcha.2012.01.003.

Ortega P R (2003) La diversidad del maíz en México. In: Sin Maíz No Hay País. G Esteva, C Marielle (eds). Consejo Nacional para las Culturas Populares: México, D.F. pp:123-154.

Ortega P R, J J Sánchez G (1989) Aportaciones al estudio de la diversidad de maíz de las partes altas de México. Rev. Fitotec. Mex. 12:105-119.

Ortiz R, S Taba, V H C Tovar, M Mezzalama, Y Xu, J Yan, J H Crouch (2009) Conserving and enhancing maize genetic resources as global public goods: A perspective from CIMMYT. Crop Sci. 50:13-28.

Perales H R, B F Benz, S B Brush (2005) Maize diversity and ethno-linguistic diversity in Chiapas, Mexico. Proc. Natl. Acad. Sci. USA 102:949-954.

Perales H R, S B Brush, C O Qualset (2003) Dynamic management of maize landraces in central Mexico. Econ. Bot. 57:21-34.

Piperno D R, K V Flannery (2001) The earliest archaeological maize (Zea mays L.) from highland Mexico: new accelerator mass spectrometry dates and their implications. Proc. Natl. Acad. Sci. USA 98: 2101-2103.

Polanco J A, T Flores M (2008) Bases para una Política de I\&D e Innovación de la Cadena de Valor del Maíz. Foro Consultivo 
Científico y Tecnológico, México D.F. 246 p.

Salinas-Moreno Y, J J Pérez-Alonso, G Vázquez-Carrillo, F AragónCuevas, G A Velázquez-Cardelas (2012) Antocianinas y actividad antioxidante en maíces (Zea mays L.) de las razas Chalqueño, Elotes Cónicos y Bolita. Agrociencia 47:815-825.

Salinas M Y, J Soria R, E T Espinosa (2010) Aprovechamiento y distribución de maíz azul en el Estado de México. Folleto Técnico 42. Instituto Nacional de Investigaciones Forestales, Agrícolas y Pecuarias. $50 \mathrm{p}$.

SIAP, Sistema de Información Agroalimentaria y Pesquera (2005) Estadística Básica Agrícola, Anuario 2005. Disponible en: www. siap.gob.mx. (Octubre 2006).

SIAP, Sistema de Información Agroalimentaria y Pesquera (2006) Estadística Básica Agrícola, Anuario 2006. Disponible en: www. siap.gob.mx. (Octubre 2006).

SIAP, Sistema de Información Agroalimentaria y Pesquera (2010) Agricultura: Producción Anual: Cierre de la producción agrícola por cultivo. Disponible en: www.siap.gob.mx/index. php?option $=$ com_wrapper\&view $=$ wrapper\&Itemid $=350 . \quad(J u-$ nio 2012).

Ureta C, E Martínez-Meyer, H R Perales, E R Álvarez-Buylla (2012) Projecting the effects of climate change on the distribution of maize races and their wild relatives in Mexico. Glob. Change Biol. 18:1073-1082.

Wellhausen E J, L M Roberts, E Hernandez X (1952) Races of Maize in Mexico. The Bussey Institution of Harvard University, Cambridge, Massachusetts. $223 \mathrm{p}$. 